\title{
CONTROLE ON-LINE POR ATRIBUTOS COM ERROS DE CLASSIFICAÇÃO: UMA ABORDAGEM ECONÔMICA COM CLASSIFICAÇÕES REPETIDAS
}

\author{
Anderson Laécio Galindo Trindade \\ Linda Lee Ho * \\ Departamento de Engenharia de Produção / EP \\ Universidade de São Paulo (USP) \\ São Paulo - SP \\ lindalee@usp.br \\ Roberto da Costa Quinino \\ Departamento de Estatística / ICEX \\ Universidade Federal de Minas Gerais (UFMG) \\ Belo Horizonte - MG \\ * Corresponding author / autor para quem as correspondências devem ser encaminhadas \\ Recebido em 03/2006; aceito em 02/2007 \\ Received March 2006; accepted February 2007
}

\begin{abstract}
Resumo
O procedimento de controle on-line de processos por atributos, proposto por Taguchi et al. (1989), consiste em amostrar um item a cada $m$ produzidos e decidir, a cada inspeção, se houve ou não a redução da fração de itens conformes produzidos. Caso o item inspecionado for não conforme, pára-se o processo para ajuste. Como o sistema de inspeção pode estar sujeito a erros de classificação, desenvolve-se um modelo probabilístico que considera classificações repetidas e independentes do item amostrado em um sistema de controle com inspeção imperfeita. Utilizando-se as propriedades de uma cadeia de Markov ergódica, obtém-se uma expressão do custo médio do sistema de controle, que pode ser minimizada por três parâmetros: o intervalo entre inspeções; o número de classificações repetidas; e o número mínimo de classificações conformes (dentre as classificações repetidas), para julgar um item como conforme. Um exemplo numérico ilustra o procedimento proposto.
\end{abstract}

Palavras-chave: controle on-line de qualidade por atributos; erros de classificação; cadeias de Markov.

\begin{abstract}
The procedure to control on-line processes for attributes proposed by Taguchi et al. (1989) consists of sampling a single item at very $m$ produced ones. If the examined item is non-conforming, the process is said to be out of control (the fraction of conforming item is reduced) and it is stopped for adjustment. However, the inspection procedure is subject to misclassification errors. So a probabilistic model was developed considering results of the repetitive and independent classifications of the examined item. Employing properties of an ergodic Markov chain, an expression of the expected cost function for the control system was obtained to be minimized by three parameters: sampling interval; number of the repetitive classifications; minor number of conforming classifications (among the repetitive classifications) to declare an item as conforming. A numerical example illustrates the proposed model.
\end{abstract}

Keywords: on-line process control for attributes; misclassification errors; Markov chain. 


\section{Introdução}

O sistema de controle on-line de processos por atributo, apresentado por Taguchi et al. (1989), considera um processo que inicia sua operação produzindo itens com uma fração conforme $p_{1}=1$ (estado I). Após a ocorrência de uma causa especial, a fração de itens conformes passa a um valor $p_{2}\left(0 \leq p_{2}<p_{1}\right)$ e o processo permanece produzindo nesta condição (estado II) até que esta mudança seja detectada e a causa especial removida. O sistema de controle consiste na inspeção periódica de um item a cada $m$ produzidos e, uma vez que este item seja julgado como não conforme, admite-se que ocorreu uma mudança da fração de itens conformes e o processo é parado para ajuste. Após o ajuste, a fração de itens conformes retorna ao valor inicial $p_{1}=1$. Exemplos em que a utilização desta metodologia é bem sucedida incluem processo automático de solda, produção de semicondutores, produção de diodos utilizados em placas de circuito impressos e em processos químicos. De maneira geral, sistemas de produção que utilizam controle automático podem beneficiar-se da metodologia aqui discutida, como apresentado por Taguchi et al. (2004).

Taguchi et al. (1989) obtém expressões analíticas para o intervalo entre inspeções $(m)$ que minimizam o custo médio do sistema de controle, porém não assumem um mecanismo explícito para a ocorrência da causa especial e tais expressões resultam de uma série de considerações e simplificações.

Nayebpour \& Woodall (1993) desenvolvem um modelo de controle para atributos incluindo um mecanismo de falha para o processo (através de uma distribuição geométrica), o que representa uma maneira formal do processo mudar do estado I para o estado II. Com isso, afirmam que não pode ser obtida uma expressão analítica para intervalo ótimo entre inspeções e a busca deste parâmetro exige o uso de métodos computacionais.

No modelo de controle para atributos apresentado por Borges et al. (2001) admite-se que o sistema de inspeção está sujeito a erros de classificação. Pode-se julgar um item conforme dado que ele não o é (erro tipo II) ou julgar um item conforme como não conforme (erro tipo I). Suas conclusões indicam que o custo do sistema de controle é sensível à presença dos erros de classificação. Porém, nos três artigos mencionados não é considerada a hipótese de classificações repetidas do item inspecionado, o que pode reduzir o impacto dos erros de classificação e, conseqüentemente, o custo médio do sistema de controle por item produzido.

O uso de classificações repetidas em um sistema de inspeção com erros de classificação não é um procedimento novo. Alguns autores já propuseram o seu uso, empregando critérios diferenciados na classificação final do item inspecionado (o item examinado é classificado repetidamente e independentemente $r$ vezes e em cada classificação, ele é avaliado em conforme ou não conforme). Em Greenberg \& Stokes (1995) este procedimento é proposto, mas admite-se apenas a existência do erro do tipo I na determinação do número ótimo de classificações repetidas. Em Quinino \& Suyama (2002), $r$ classificações repetidas são realizadas no item inspecionado, que é declarado conforme se pelo menos forem observadas $a$ classificações conformes. Os parâmetros $a$ e $r$ são determinados segundo uma abordagem econômica. Em Quinino \& Ho (2004), os itens são examinados repetidamente até observar $a$ classificações conformes ou $b$ classificações não conformes (no primeiro caso, o item é declarado conforme e no segundo critério, o item é declarado não conforme). Para outros resultados, o procedimento de classificações repetidas continua. O objetivo foi determinar os parâmetros $a$ e $b$ ótimos também segundo uma abordagem econômica. Porém, tais trabalhos não consideram a mudança da fração de itens conformes durante a produção. 
Este trabalho tem por objetivo desenvolver um modelo probabilístico que considera as classificações repetidas e independentes do item inspecionado através de cadeias de Markov de estados discretos para determinar uma estratégia ótima de controle (on-line por atributos) em um processo cujo sistema de inspeção está sujeito a erros de classificação. A estratégia ótima consiste na determinação do intervalo entre inspeções $(m)$, do número de classificações repetidas do item inspecionado $(r)$ e do número mínimo de classificações conformes (dentre as $r$ ) para julgar o item conforme $(w)$ que minimizam o custo médio do sistema de controle. Este artigo está estruturado do seguinte modo: a Seção 2 apresenta o modelo probabilístico proposto. O custo médio do sistema de controle é obtido na Seção 3. Na Seção 4, um exemplo numérico ilustra o efeito da presença dos erros de classificação e das classificações repetidas sobre o custo médio do sistema de controle. As conclusões e sugestões são apresentadas na Seção 5.

\section{Modelo Probabilístico}

Considere que itens são produzidos em um processo que, no estado I, possui uma fração de itens conformes $p_{1}$ e, no estado II, a fração de itens conformes diminua para $p_{2}$, com $0 \leq p_{2}<p_{1} \leq 1$. A probabilidade de mudança do estado I para o estado II, a cada item produzido, é dada por uma distribuição geométrica com parâmetro $\pi$, sendo $0<\pi<1$. $\mathrm{O}$ sistema de controle consiste em inspecionar o $m$-ésimo item a cada ciclo de $m$ produzidos (aqui denominado ciclo de inspeção). O item inspecionado é submetido a $r$ classificações (em cada classificação, o item é avaliado em conforme ou não conforme) e caso apresente pelo menos $w$ classificações conforme, o item é julgado conforme. Como o sistema de inspeção é considerado imperfeito, cada classificação a que o item é submetido está sujeita a dois tipos de erro: classificar um item conforme como não conforme $(\alpha)$, e classificar um item não conforme como conforme $(\beta)$. Caso o item inspecionado seja julgado não conforme, admite-se que ocorreu a mudança de estado e o processo de produção é interrompido, ajustado, e reiniciado sob o estado I. Entre o instante em que o item é inspecionado e o instante em que o processo é parado podem ser produzidos $L$ itens, aos quais se denomina atraso. O processo produzindo sob o estado II só pode retornar ao estado I após a ocorrência de um ajuste.

Conforme a descrição, o processo de inspeção pode ser modelado como uma cadeia de Markov estacionária considerando o espaço de estados discretos $E=\left\{s_{0} k_{1}, s_{0} k_{0}, s_{1} k_{1}, s_{1} k_{0}, s_{2} k_{1}\right.$, $\left.s_{2} k_{0}\right\}$. A variável $s$ indica o real estado do processo em que os itens que compõem o ciclo de inspeção foram produzidos. Quando $s=O\left(s_{0}\right)$, todos os itens (inclusive o inspecionado) foram produzidos no estado I. Quando $s=2\left(s_{2}\right)$ todos os itens do ciclo (inclusive o inspecionado) foram produzidos no estado II. Quando $s=1\left(s_{1}\right)$ necessariamente ocorreu uma mudança do estado I para o estado II no ciclo considerado e pelo menos o item inspecionado foi produzido no estado II. A variável $k$, por sua vez, indica se o item inspecionado foi declarado conforme $\left(k_{1}\right)$ ou não conforme $\left(k_{0}\right)$. A Figura 1 relaciona o processo de produção aos estados da cadeia. A matriz de transição $\mathbf{P}$ em (1) apresenta as probabilidades de migração entre estes estados em ciclos subseqüentes de inspeção. 


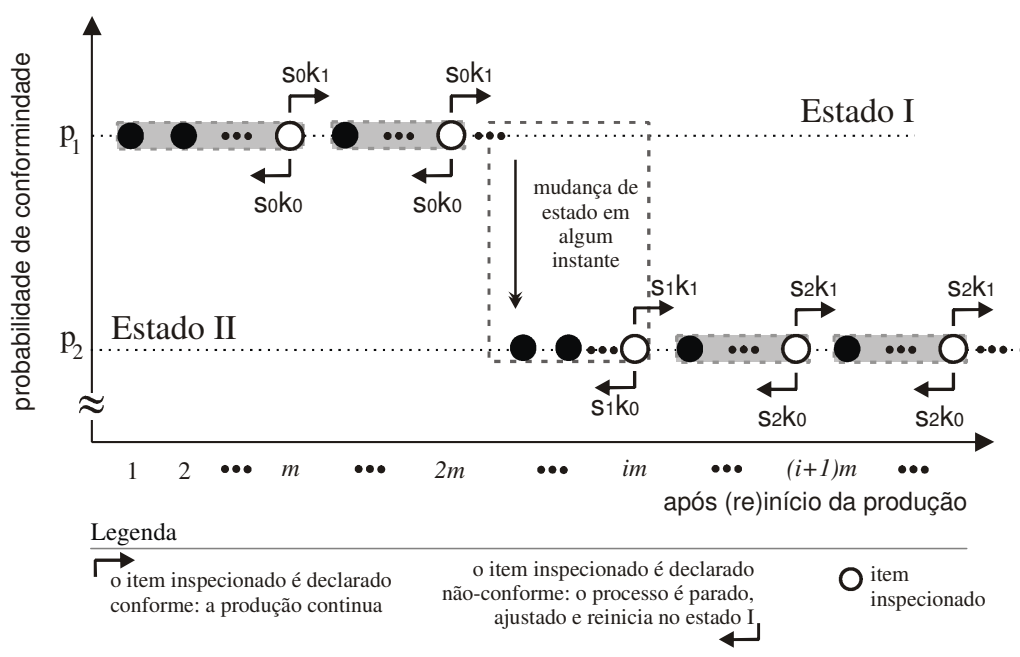

Figura 1 - Fluxograma do processo e sistema de controle.

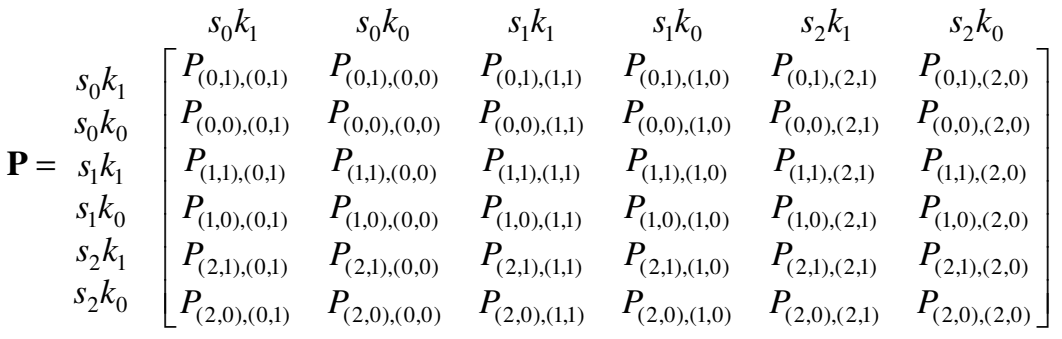

Como exemplo para ilustrar a notação utilizada, $P_{(2,1),(0,0)}$ representa a probabilidade de que a inspeção $i$ ocorra sob o estado da cadeia $s_{2} k_{1}$ e que a inspeção $(i+1)$ ocorra sob o estado $s_{0} k_{0}$, ou simplesmente $\operatorname{Pr}\left(E_{i+1}=s_{0} k_{0} \mid E_{i}=s_{2} k_{1}\right)$. A seguir estão detalhadas as probabilidades de transição dadas em (1).

A probabilidade $P_{(0,1),(0,1)}, \operatorname{Pr}\left(E_{i+1}=s_{0} k_{1} \mid E_{i}=s_{0} k_{1}\right)$, é o produto das probabilidades: a) de todos os $m$ itens do ciclo serem produzidos no estado I, e b) do item inspecionado ser julgado conforme.

Para obter a) é necessário definir uma variável aleatória não observável, $\Theta_{i}$, com $i \geq 0$, que representa a probabilidade de produção de um item conforme no instante em que é produzido o $i$-ésimo item. Com isso, a probabilidade de que o processo permaneça sob o estado I até o $m$-ésimo item produzido é

$$
\operatorname{Pr}\left\{\Theta_{m}=p_{1}\right\}=\operatorname{Pr}\left\{\Theta_{1}=p_{1}, \ldots, \Theta_{m}=p_{1}\right\}=(1-\pi)^{m}
$$

Devido à existência de erros de classificação, a probabilidade de que a inspeção declare um item conforme é a soma de duas probabilidades: i) probabilidade de julgar um item conforme como conforme e ii) probabilidade de julgar um item não conforme como conforme. Julgar um item conforme, classificando-o repetidamente e independentemente é um processo binomial em que a probabilidade de sucesso é dada por (1- $\alpha)$ caso, o item seja de fato conforme ou $\beta$ caso o item seja, de fato, não conforme. Sendo $Y$ uma variável aleatória que representa $o$ 
número de vezes que o item inspecionado é classificado como conforme e $\theta$ uma variável aleatória que define o estado real do item $(\theta=1$, item conforme; $\theta=0$, item não conforme) tem-se:

$$
\begin{gathered}
\operatorname{Pr}\{Y \geq w \mid \theta=1\}=1-B_{w-1}(r, 1-\alpha) \\
\operatorname{Pr}\{Y \geq w \mid \theta=0\}=1-B_{w-1}(r, \beta)
\end{gathered}
$$

em que $B_{x}(n, p)$ denota a probabilidade binomial acumulada até $x$ sucessos em $n$ tentativas, sendo $p$ a probabilidade de sucesso. Note que (3) representa a probabilidade de uma decisão correta, que pode ser denotada como a probabilidade complementar de um erro $\alpha^{*}$, enquanto (4) corresponde à probabilidade de uma decisão errada sobre o julgamento do item inspecionado que será denotado como um erro $\beta^{*}$. Assim

$$
\begin{gathered}
1-\alpha^{*}=1-B_{w-1}(r, 1-\alpha) \therefore \alpha^{*}=B_{w-1}(r, 1-\alpha) \\
\beta^{*}=1-B_{w-1}(r, \beta)
\end{gathered}
$$

Combinando (2), (3) e (4), tem-se:

$$
\begin{aligned}
P_{(0,1),(0,1)} & =\operatorname{Pr}\left\{\Theta_{m}=p_{1}\right\}\left\{p_{1} \operatorname{Pr}\{Y \geq w \mid \theta=1\}+\left(1-p_{1}\right) \operatorname{Pr}\{Y \geq w \mid \theta=0\}\right\} \\
& =(1-\pi)^{m}\left\{p_{1}\left(1-\alpha^{*}\right)+\left(1-p_{1}\right) \beta^{*}\right\} .
\end{aligned}
$$

A probabilidade $P_{(0,1),(0,0)}$, por sua vez, implica em julgar o item inspecionado como não conforme, mesmo com todos os itens produzidos no ciclo sob o estado I. Portanto,

$$
\begin{aligned}
P_{(0,1),(0,0)} & =\operatorname{Pr}\left\{\Theta_{m}=p_{1}\right\}\left\{p_{1} \operatorname{Pr}\{Y<w \mid \theta=1\}+\left(1-p_{1}\right) \operatorname{Pr}\{Y<w \mid \theta=0\}\right\} \\
& =(1-\pi)^{m}\left\{p_{1} \alpha^{*}+\left(1-p_{1}\right)\left(1-\beta^{*}\right)\right\} .
\end{aligned}
$$

A probabilidade $P_{(0,1),(1,1)}, \operatorname{Pr}\left(E_{i+1}=s_{1} k_{1} \mid E_{i}=s_{0} k_{1}\right)$, consiste na mudança do estado I para o estado II desde a última inspeção, bem como no julgamento do item inspecionado como conforme. Note que agora a probabilidade do item inspecionado ser conforme é dada por $p_{2}$. Com isso, tem-se:

$$
\begin{aligned}
P_{(0,1),(1,1)} & =\left(1-\operatorname{Pr}\left\{\Theta_{m}=p_{1}\right\}\right)\left\{p_{2} \operatorname{Pr}\{Y \geq w \mid \theta=1\}+\left(1-p_{2}\right) \operatorname{Pr}\{Y \geq w \mid \theta=0\}\right\} \\
& =\left[1-(1-\pi)^{m}\right]\left\{p_{2}\left(1-\alpha^{*}\right)+\left(1-p_{2}\right) \beta^{*}\right\} .
\end{aligned}
$$

A probabilidade $P_{(0,1),(1,0)}, \operatorname{Pr}\left(E_{i+1}=s_{1} k_{0} \mid E_{i}=s_{0} k_{1}\right)$, indica que há mudança do estado do processo $(\mathrm{I} \rightarrow \mathrm{II})$, sendo o item inspecionado declarado não conforme.

$$
\begin{aligned}
P_{(0,1),(1,0)} & =\left(1-\operatorname{Pr}\left\{\Theta_{m}=p_{1}\right\}\right)\left\{p_{2} \operatorname{Pr}\{Y<w \mid \theta=1\}+\left(1-p_{2}\right) \operatorname{Pr}\{Y<w \mid \theta=0\}\right\} \\
& =\left[1-(1-\pi)^{m}\right]\left\{p_{2} \alpha^{*}+\left(1-p_{2}\right)\left(1-\beta^{*}\right)\right\} .
\end{aligned}
$$

As probabilidades restantes, $P_{(1,1),(2,1)}, P_{(1,1),(2,0)}, P_{(2,1),(2,1)}$ e $P_{(2,1),(2,0)}$, devem considerar apenas a probabilidade de julgar o item inspecionado conforme ou não conforme, pois o ciclo já se inicia no estado de produção II.

$$
\begin{aligned}
P_{(1,1),(2,1)} & =P_{(2,1)(2,1)}=\left\{p_{2} \operatorname{Pr}\{Y \geq w \mid \theta=1\}+\left(1-p_{2}\right) \operatorname{Pr}\{Y \geq w \mid \theta=0\}\right\} \\
& =\left\{p_{2}\left(1-\alpha^{*}\right)+\left(1-p_{2}\right) \beta^{*}\right\} \\
P_{(1,1),(2,0)} & =P_{(2,1)(2,0)}=\left\{p_{2} \operatorname{Pr}\{Y<w \mid \theta=1\}+\left(1-p_{2}\right) \operatorname{Pr}\{Y<w \mid \theta=0\}\right\} \\
& =\left\{p_{2} \alpha^{*}+\left(1-p_{2}\right)\left(1-\beta^{*}\right)\right\} .
\end{aligned}
$$


As probabilidades $P_{(0,1),(2,1)}=P_{(0,1),(2,0)}=0$. Em todos os estados nos quais há ajuste do processo, um novo ciclo de inspeções é iniciado necessariamente sob o estado I. Portanto, todas as linhas pares da matriz $\mathbf{P}$ são iguais à primeira linha da matriz $\mathbf{P}$. Como o processo só retorna ao estado de produção I após um ajuste, as probabilidades $P_{(1,1),(0,1)}=P_{(1,1),(0,0)}=$ $P_{(2,1),(0,1)}=P_{(2,1),(0,0)}=0$. Por fim, $P_{(1,1),(1,1)}=P_{(1,1),(1,0)}=P_{(2,1),(1,1)}=P_{(2,1),(1,0)}=0$. A matriz $\mathbf{P}$ pode então ser reescrita como

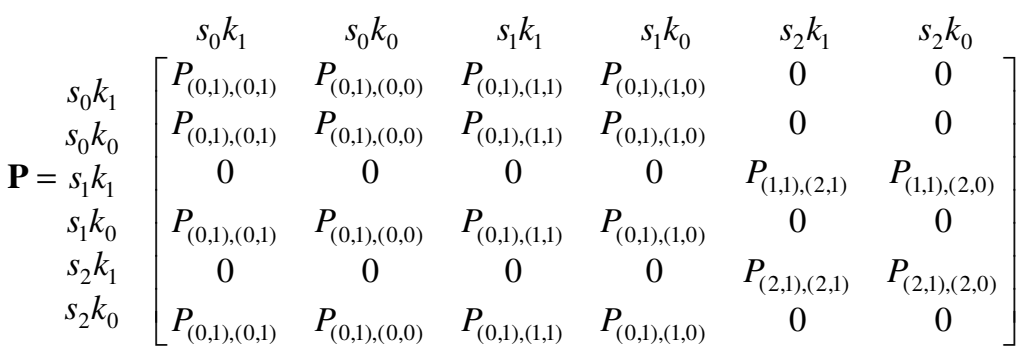

Como a matriz $\mathbf{P}$ é uma matriz de uma cadeia regular (detalhes em Ross, 2003), então existe o $\lim _{j \rightarrow \infty} \mathbf{P}^{(j)}=\mathbf{Y}$, em que todas as linhas da matriz $\mathbf{Y}$ são iguais ao vetor linha $\mathbf{y}=\left[\begin{array}{llll}y_{(0,1)} & y_{(0,0)} & \ldots & y_{(2,0)}\end{array}\right]$. O vetor $\mathbf{y}$ é um vetor de probabilidade $\left(\Sigma_{i} y_{i}=1\right)$ no estado estacionário, com todos os valores $y_{i}$ estritamente positivos. Cada elemento de y é interpretado como sendo a fração do número de inspeções que são realizadas em cada um dos estados da cadeia após um número suficientemente grande de inspeções. Como $\mathbf{P}^{(j+l)}=\mathbf{P}^{(j)} \mathbf{P}$ e $\lim _{j \rightarrow \infty} \mathbf{P}^{(j+l)}=\lim _{j \rightarrow \infty} \mathbf{P}^{(j)}=\mathbf{Y}$, então no limite a equação $\mathbf{Y}=\mathbf{Y} \mathbf{P}$ é verdadeira. Como todas as linhas de $\mathbf{Y}$ são iguais à $\mathbf{y}$, a equação $\mathbf{y}=\mathbf{y P}$ também é válida no limite, e pode ser escrita como

$$
\mathbf{y}=\mathbf{y P} \therefore \mathbf{y}(\mathbf{P}-\mathbf{I})=\mathbf{0}
$$

em que I é a matriz identidade. Portanto, o vetor $\mathbf{y}$ pode ser obtido a partir da resolução do sistema linear (5) com a restrição de que $\Sigma_{i} y_{i}=1$. Resolvendo (5), os elementos de y são dados por

$$
\begin{gathered}
y_{(0,1)}=\frac{P_{(0,1),(0,0)} P_{(1,1),(2,0)}}{P_{(0,1),(1,1)}+P_{(1,1),(2,0)}} \\
y_{(0,0)}=\frac{P_{(0,1),(0,1)} P_{(1,1),(2,0)}}{P_{(0,1),(1,1)}+P_{(1,1),(2,0)}} \\
y_{(1,1)}=y_{(2,0)}=\frac{P_{(0,1),(1,1)} P_{(1,1),(2,0)}}{P_{(0,1),(1,1)}+P_{(1,1),(2,0)}} \\
y_{(1,0)}=\frac{P_{(0,1),(1,0)} P_{(1,1),(2,0)}}{P_{(0,1),(1,1)}+P_{(1,1),(2,0)}} \\
y_{(2,1)}=\frac{P_{(0,1),(1,1)}\left(1-P_{(1,1),(2,0)}\right)}{P_{(0,1),(1,1)}+P_{(1,1),(2,0)}} .
\end{gathered}
$$




\section{Custo Médio do Sistema de Controle}

Para obter a função custo, são necessárias algumas considerações adicionais. Tão logo o item inspecionado é declarado não conforme, o processo é parado para ajuste. Os itens produzidos, entre o instante em que o item inspecionado é amostrado (e declarado não conforme) e a completa parada do processo, são denominados atraso $(L)$ e são descartados. O item inspecionado é classificado $r$ vezes e inutilizado após a inspeção (por exemplo, pequenas partes do item inspecionado podem ser retiradas).

Considere os custos:

- $c_{0}$ - custo de uma classificação do item inspecionado;

- $c_{1}$ - custo de enviar um item não conforme para o mercado ou próximas etapas do processo;

- $c_{2}$ - custo de ajuste do processo;

- $c_{3}$ - custo de eliminar um item não conforme;

- $c_{4}$ - custo de eliminar um item conforme.

Os componentes $c_{3}$ e $c_{4}$ são utilizados para a eventualidade dos itens descartados serem submetidos a algum processo de reaproveitamento, no qual o custo de reaproveitamento de um item conforme pode diferir daquele não conforme. Caso isso não seja possível, basta estabelecer $c_{3}=c_{4}$, custo este que deve ser o custo unitário de produção (desconsiderado o sistema de controle).

O custo de cada estado $(s, k)$ pode ser escrito como $V_{(s, k)}=r c_{0}+\xi_{(s, k)}+\eta_{(s, k)}+\varphi_{(s, k)}$, em que

- $r c_{0}$ é o custo de realizar $r$ classificações do item inspecionado (presente em todos os estados da cadeia);

- $\xi_{(s, k)}$, custo de enviar itens defeituosos para o consumidor ou estágios posteriores do processo;

- $\eta_{(s, k)}$, custo relacionado ao descarte do item inspecionado;

- $\varphi_{(s, k)}$, custo de ajuste e de descartar os itens que compõem o atraso.

A seguir estão detalhados os custos para os estados da cadeia de Markov. Para o estado $\left(s_{0} k_{1}\right)$, sabe-se que todos os $m$ itens do ciclo foram produzidos no estado I. Dos $(m-1)$ itens que são enviados para o mercado, $(m-1)\left(1-p_{1}\right)$ é o número esperado de itens não-conformes. Portanto

$$
\xi(0,1)=c_{1}(m-1)\left(1-p_{1}\right) .
$$

O item inspecionado foi declarado conforme, mas, devido à existência de erros de classificação, há uma probabilidade de que ele seja não conforme. Assim

$$
\eta(0,1)=c_{3} \frac{\left(1-p_{1}\right) \beta^{*}}{p_{1}\left(1-\alpha^{*}\right)+\left(1-p_{1}\right) \beta^{*}}+c_{4} \frac{p_{1}\left(1-\alpha^{*}\right)}{p_{1}\left(1-\alpha^{*}\right)+\left(1-p_{1}\right) \beta^{*}} .
$$

Como não há ajuste do processo então $\varphi_{(0,1)}=0$.

Para o estado $\left(s_{0} k_{0}\right)$, todos os $m$ itens do ciclo também são produzidos no estado I, logo $\xi_{(0,0)}=\xi_{(0,1)}$. Já o item inspecionado foi declarado não conforme, e o custo de seu descarte é

$$
\eta(0,0)=c_{3} \frac{\left(1-p_{1}\right)\left(1-\beta^{*}\right)}{p_{1} \alpha^{*}+\left(1-p_{1}\right)\left(1-\beta^{*}\right)}+c_{4} \frac{p_{1} \alpha^{*}}{p_{1} \alpha^{*}+\left(1-p_{1}\right)\left(1-\beta^{*}\right)} .
$$


Neste estado há ajuste do processo e $L$ itens que compõem o atraso são descartados. Apesar do item inspecionado ter sido produzido no estado I, há uma probabilidade não nula de ocorrer a mudança de estado em algum dos itens que compõe o atraso, o que é considerado em

$$
\begin{aligned}
\varphi(0,0)= & c_{2}+L(1-\pi)^{L}\left[\left(1-p_{1}\right) c_{3}+p_{1} c_{4}\right. \\
& +c_{3} \sum_{i=1}^{L}(1-\pi)^{i-1} \pi\left[(i-1)\left(1-p_{1}\right)+(L-i+1)\left(1-p_{2}\right)\right] \\
& +c_{4} \sum_{i=1}^{L}(1-\pi)^{i-1} \pi\left[(i-1) p_{1}+(L-i+1)\left(1-p_{2}\right)\right] \\
= & c_{2}+L(1-\pi)^{L}\left[\left(1-p_{1}\right) c_{3}+p_{1} c_{4}\right. \\
& +\frac{(1-\pi)\left(1-(1-\pi)^{L}\right)-(1-\pi)^{L} \pi L}{\pi}\left[\left(1-p_{1}\right) c_{3}+p_{1} c_{4}\right] \\
& +\frac{(1-\pi)^{L+1}+\pi(L+1)-1}{\pi}\left[\left(1-p_{2}\right) c_{3}+p_{1} c_{4}\right]
\end{aligned}
$$

onde $i$ é o instante em que o primeiro item é produzido no estado II.

Para o estado $\left(s_{1} k_{1}\right)$, uma parte dos $m$ itens é produzida no estado I. Assim

$$
\begin{aligned}
& \varepsilon(1,1)= c_{1} \sum_{i=1}^{m} \frac{\pi(1-\pi)^{-i-1}}{1-(1-\pi)^{m}}\left[(i-1)\left(1-p_{1}\right)+(m-i)\left(1-p_{2}\right)\right] \\
&= c_{1}\left(1-p_{1}\right) \frac{(1-\pi)\left(1-(1-\pi)^{m}\right)-\pi m(1-\pi)^{m}}{\pi\left(1-(1-\pi)^{m}\right)} \\
&+c_{1}\left(1-p_{2}\right) \frac{\pi m+(1-\pi)^{m}-1}{\pi\left(1-(1-\pi)^{m}\right)} . \\
& \eta(1,1)=c_{3} \frac{\left(1-p_{2}\right) \beta^{*}}{p_{2}\left(1-\alpha^{*}\right)+\left(1-p_{2}\right) \beta^{*}}+c_{4} \frac{p_{2}\left(1-\alpha^{*}\right)}{p_{2}\left(1-\alpha^{*}\right)+\left(1-p_{2}\right) \beta^{*}} .
\end{aligned}
$$

Como não há ajuste do processo segue que $\varphi_{(1,1)}=0$.

Para o estado $\left(s_{1} k_{0}\right), \xi_{(1,0)}=\xi_{(1,1)}$. O custo associado a descartar o item inspecionado é

$$
\eta(1,0)=c_{3} \frac{\left(1-p_{2}\right)\left(1-\beta^{*}\right)}{p_{2} \alpha^{*}+\left(1-p_{2}\right)\left(1-\beta^{*}\right)}+c_{4} \frac{p_{2} \alpha^{*}}{p_{2} \alpha^{*}+\left(1-p_{2}\right)\left(1-\beta^{*}\right)} .
$$

Como a mudança para o estado II já ocorreu em algum ponto do ciclo em questão, todos os itens que compõem o atraso são produzidos no estado II. Assim

$$
\varphi(1,0)=c_{2}+L\left[c_{3}\left(1-p_{2}\right)+c_{4} p_{2}\right] .
$$

Para o estado $\left(s_{2} k_{1}\right)$, todos os itens do ciclo são produzidos no estado II. Assim

$$
\xi(2,1)=c_{1}(m-1)\left(1-p_{2}\right) .
$$

Já o custo de descartar o item inspecionado $\eta_{(2,1)}=\eta_{(1,1)}$ e como não há ajuste, $\varphi_{(2,1)}=0$. 
Para o estado $\left(s_{2} k_{0}\right)$, são válidas as igualdades de custo: $\xi_{(2,0)}=\xi_{(2,1)}, \eta_{(2,0)}=\eta_{(1,0)}$ e $\varphi_{(2,0)}=\varphi_{(1,0)}$.

Em cada ciclo de inspeção, $(m-1)$ itens são enviados para o mercado ou para os próximos estágios de produção. Para um número suficientemente grande de inspeções realizadas, $\mathbf{y}$ corresponde ao vetor de probabilidade de ocorrência de cada estado da cadeia. Portanto, o custo médio por item produzido e não descartado (em cada ciclo de inspeção) é:

$$
C(m, r, w)=\frac{1}{m-1}\left(r c_{0}+\sum_{i=0}^{2} \sum_{j=0}^{1} y\left(s_{i}, k_{j}\right)\left[\xi\left(s_{i}, k_{j}\right)+\eta\left(s_{i}, k_{j}\right)+\varphi\left(s_{i}, k_{j}\right)\right) .\right.
$$

O problema consiste em determinar $m, r$ e $w$ que minimizam (6), ou seja

$$
\left(m^{0}, r^{0}, w^{0}\right)=\arg \min _{(m, r, w)} C
$$

Como não é possível obter uma expressão analítica para (7), utiliza-se uma busca computacional exaustiva para determinação dos parâmetros ótimos. As versões do programa em Microsoft Excel e Matlab podem ser obtidas no endereço www.est.ufmg.br/ roberto. A seguir está um exemplo numérico para ilustrar o procedimento proposto.

\section{Exemplos Numéricos}

Para ilustrar o modelo proposto com um exemplo numérico, considere um processo de manufatura com os seguintes custos:

- Custo de inspeção $c_{0}=\$ 0,25$;

- Custo de envio de um item não conforme $c_{1}=\$ 20$;

- Custo de ajuste $c_{2}=\$ 100$;

- Custo de eliminar um item conforme ou não conforme iguais, $c_{3}=c_{4}=\$ 2,0$.

E os seguintes parâmetros de processo:

- Fração conforme no estado I: $p_{1}=0,99$;

- Fração conforme no estado II: $p_{2}=0,80$;

- Probabilidade de mudança do estado I para estado II: $\pi=0,0001$;

- Erros de classificação: $\alpha=\beta=0,01$ e

- O número de itens produzidos entre a sinalização (item não conforme) e a completa parada do processo (atraso) $L=10$.

sendo que os custos estão expressos em unidades monetárias. Para simplificar a execução do modelo, considere que o item será julgado conforme caso mais da metade das $r$ classificações sejam conformes, ou seja, $w=\lceil 0,5 r\rceil$. A Figura 2 mostra o custo médio do sistema de controle por item produzido (e não descartado) em função do intervalo entre inspeções $(m)$ e do número de classificações repetidas que o item inspecionado é submetido $(r)$. Os valores ótimos obtidos são $m^{\circ}=51$ e $r^{\circ}=3$, que resultam em um custo médio de $\$ 0,3762$. Caso uma única classificação do item inspecionado seja realizada $(r=1)$, o intervalo ótimo é $m^{\circ}=55$ e o custo médio $\$ 0,3853$, (2,42\% maior que aquele com múltiplas classificações). 


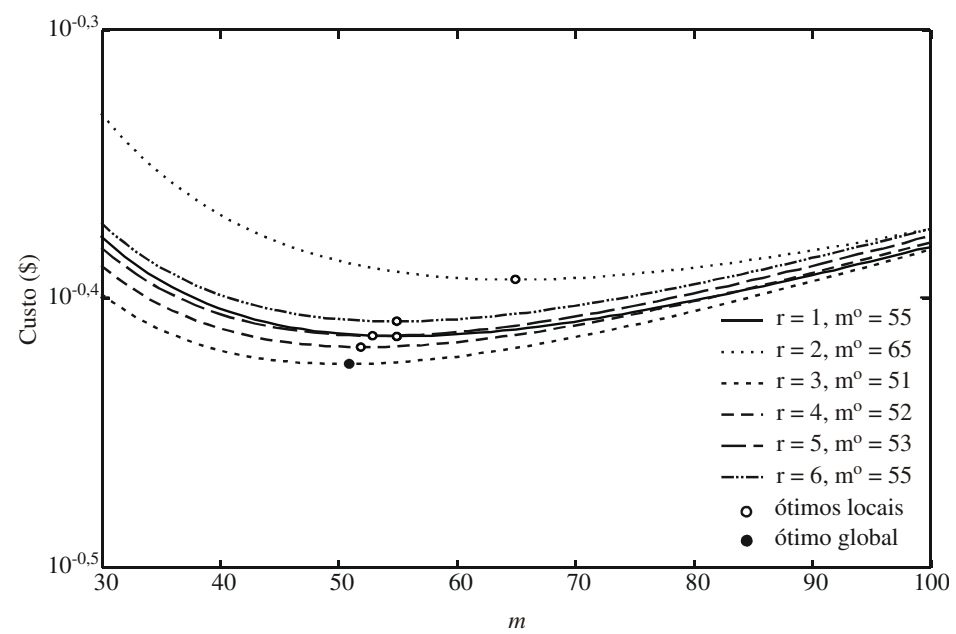

Figura 2 - Gráfico de custo médio $(C)$ em função de $m$ e $r$.

Considere agora que os erros Tipo I e II são desprezados durante a determinação da estratégia ótima de controle. Para esta situação, tem-se $m^{\circ}=47$ e $r^{\circ}=1$. Porém, adotar esta estratégia em um sistema de inspeção que possui tais erros, sendo $\alpha=\beta=1 \%$, gera um custo médio real $C=\$ 0,3876$. Este custo é $0,60 \%$ maior que o custo da estratégia com $r=1$ (e 3,03\% maior que aquela obtida considerando classificações repetidas).

O critério $w=\lceil 0,5 r\rceil$ é uma restrição a um parâmetro que também pode ser utilizado para minimizar o custo médio. Sem esta imposição, a estratégia ótima torna-se $m^{\circ}=48, r^{\circ}=2$ e $w^{\circ}=1$, e o custo médio $C=\$ 0,3719,(1,14 \%$ menor que melhor estratégia obtida até então).

A Tabela 1 mostra os valores ótimos para $m, r$ e $w$ variando-se apenas um parâmetro por vez, com os demais mantidos constantes nos valores utilizados no exemplo numérico do início desta seção. Apesar da faixa de variação escolhida ser arbitrária, respeitam-se as seguintes condições que tornam as combinações utilizadas razoáveis:

- a fração de itens conformes no estado II é inferior à do estado I $\left(p_{2}<p_{1}\right)$;

- o custo de descartar um item conforme é o mesmo de um item não conforme $\left(c_{3}=c_{4}\right)$;

- o custo de ajuste é muito maior que o custo de inspeção $\left(c_{2} \gg c_{0}\right) \mathrm{e}$

- o custo de enviar um item não conforme para o mercado é maior que o custo de descartá-lo $\left(c_{1}>c_{3}\right)$.

Os parâmetros $r^{\mathrm{O}}$ e $w^{\mathrm{o}}$ mostram-se insensíveis aos custos $c_{1}, c_{2}$ e $c_{3}$ nas faixas utilizadas, enquanto o aumento do custo de cada classificação $\left(c_{0}\right)$ leva a uma redução do número de classificações a que o item inspecionado é submetido, como esperado.

Já o parâmetro $m^{\circ}$ apresenta um comportamento distinto para cada um dos custos avaliados. Um aumento no custo de inspeção $\left(c_{0}\right)$ ou no custo de ajuste $\left(c_{2}\right)$ leva ao aumento de $m^{\circ}$, reduzindo a freqüência das inspeções e a ocorrência de ajustes desnecessários (ajustar o processo que opera no estado I). Um aumento no custo de envio de itens defeituosos para o mercado $\left(c_{1}\right)$ leva à redução do intervalo entre inspeções, ou seja, ao aumento na freqüência das inspeções, com o objetivo de detectar mais prontamente a ocorrência da mudança de estado do processo. Para a faixa pesquisada, $c_{3}$ não altera a estratégia ótima de inspeção. 
Uma redução na fração de itens conformes produzidos no estado I $\left(p_{1}\right)$ leva a um aumento no intervalo entre inspeções. Isso ocorre porque, com mais itens não-conformes sendo produzidos no estado I, aumenta a ocorrência de ajustes desnecessários, pois mais itens não-conformes são amostrados. Já o aumento na fração de itens conformes produzidos no estado II $\left(p_{2}\right)$ reduz a probabilidade de detectar a ocorrência da mudança, pois mais itens conformes são amostrados. Como resposta a isso, as inspeções tornam-se mais freqüentes, ou seja, há uma redução no valor de $m^{\circ}$. Estes comportamentos ficam claros quando o objetivo do sistema de controle é revisto: detectar a mudança de estado do processo tão logo ela ocorra, sendo $\pi$ o parâmetro que controla a mudança do processo. Como mostra a Tabela 1, o aumento deste parâmetro $(\pi)$ leva à redução no intervalo entre inspeções, pois em média a mudança de estado $(\mathrm{I} \rightarrow \mathrm{II})$ passa a ocorrer após um menor número de itens produzidos pelo processo.

O uso de classificações repetidas $(r)$ e de um critério para declarar um item conforme $(w)$ atuam em resposta à existência dos erros Tipo I $(\alpha)$ e Tipo II $(\beta)$ no sistema de inspeção. Um aumento de $\alpha$ eleva a probabilidade de declarar um item conforme como não conforme. A estratégia ótima contrapõe este aumento exigindo, proporcionalmente, que um número menor de classificações resulte em conformidade para julgar o item conforme. Para $\alpha=1 \%$, pelo menos $w^{\circ} / r^{\circ}=50 \%$ das classificações devem ser conformes para julgar o item conforme. Quando $\alpha=10 \%$, a razão diminui para $w^{\circ} / r^{\circ}=33,3 \%$. Comportamento oposto é observado com o aumento de $\beta$, classificar um item defeituoso como conforme. Quando $\beta=1 \%$, no mínimo $w^{\circ} / r^{\circ}=50 \%$ das classificações devem ser conformes para julgar o item conforme. Quando $\beta=10 \%$ este valor passa a $w^{\circ} / r^{\circ}=66,6 \%$ devido ao maior número de falsas conformidades.

Tabela 1 - Determinação de $m^{\circ}, r^{\circ}, w^{\circ}$ e C para uma variação individual dos parâmetros do modelo, os demais constantes mantidos nos valores utilizados no exemplo numérico.

\begin{tabular}{ccccc|ccccc|ccccc}
\hline $\boldsymbol{\pi \times 1 \mathbf { 1 } ^ { \mathbf { 3 } }}$ & $\boldsymbol{C}$ & $\boldsymbol{m}^{\mathbf{0}}$ & $\boldsymbol{r}^{\mathbf{0}}$ & $\boldsymbol{w}^{\mathbf{0}}$ & $\boldsymbol{p}_{\mathbf{1}}$ & $\boldsymbol{C}$ & $\boldsymbol{m}^{\mathbf{0}}$ & $\boldsymbol{r}^{\mathbf{0}}$ & $\boldsymbol{w}^{\mathbf{0}}$ & $\boldsymbol{p}_{\mathbf{2}}$ & $\boldsymbol{C}$ & $\boldsymbol{m}^{\mathbf{0}}$ & $\boldsymbol{r}^{\mathbf{0}}$ & $\boldsymbol{w}^{\mathbf{0}}$ \\
\hline $\mathbf{0 , 1 0 0}$ & 0,3719 & 48 & 2 & 1 & 0,850 & 3,1855 & 245 & 1 & 1 & 0,200 & 0,3474 & 56 & 2 & 1 \\
0,325 & 0,5262 & 28 & 2 & 1 & 0,885 & 2,5221 & 155 & 2 & 1 & 0,350 & 0,3551 & 53 & 2 & 1 \\
0,550 & 0,6383 & 22 & 2 & 1 & 0,920 & 1,8319 & 110 & 2 & 1 & 0,500 & 0,3621 & 51 & 2 & 1 \\
0,775 & 0,7334 & 19 & 2 & 1 & 0,955 & 1,1178 & 78 & 2 & 1 & 0,650 & 0,3681 & 49 & 2 & 1 \\
1,000 & 0,8186 & 17 & 2 & 1 & $\mathbf{0 , 9 9 0}$ & 0,3719 & 48 & 2 & 1 & $\mathbf{0 , 8 0 0}$ & 0,3719 & 48 & 2 & 1 \\
\hline $\boldsymbol{\alpha}$ & $\boldsymbol{C}$ & $\boldsymbol{m}^{\mathbf{0}}$ & $\boldsymbol{r}^{\mathbf{0}}$ & $\boldsymbol{w}^{\mathbf{0}}$ & $\boldsymbol{\beta}$ & $\boldsymbol{C}$ & $\boldsymbol{m}^{\mathbf{0}}$ & $\boldsymbol{r}^{\mathbf{0}}$ & $\boldsymbol{w}^{\mathbf{0}}$ & $\boldsymbol{c}_{\mathbf{0}}$ & $\boldsymbol{C}$ & $\boldsymbol{m}^{\mathbf{0}}$ & $\boldsymbol{r}^{\mathbf{0}}$ & $\boldsymbol{w}^{\mathbf{0}}$ \\
\hline $\mathbf{0 , 0 1 0}$ & 0,3719 & 48 & 2 & 1 & $\mathbf{0 , 0 1 0}$ & 0,3719 & 48 & 2 & 1 & $\mathbf{0 , 2 5 0}$ & 0,3719 & 48 & 2 & 1 \\
0,033 & 0,3739 & 49 & 2 & 1 & 0,033 & 0,3749 & 47 & 2 & 1 & 0,563 & 0,3847 & 52 & 2 & 1 \\
0,055 & 0,3779 & 51 & 2 & 1 & 0,055 & 0,3768 & 50 & 3 & 2 & 0,875 & 0,3965 & 59 & 1 & 1 \\
0,078 & 0,3786 & 50 & 3 & 1 & 0,078 & 0,3774 & 50 & 3 & 2 & 1,188 & 0,4018 & 61 & 1 & 1 \\
0,100 & 0,3796 & 50 & 3 & 1 & 0,100 & 0,3781 & 50 & 3 & 2 & 1,500 & 0,4070 & 62 & 1 & 1 \\
\hline $\boldsymbol{c}_{\mathbf{1}}$ & $\boldsymbol{C}$ & $\boldsymbol{m}^{\mathbf{0}}$ & $\boldsymbol{r}^{\mathbf{0}}$ & $\boldsymbol{w}^{\mathbf{0}}$ & $\mathbf{c _ { 2 }}$ & $\boldsymbol{C}$ & $\boldsymbol{m}^{\mathbf{0}}$ & $\boldsymbol{r}^{\mathbf{0}}$ & $\boldsymbol{w}^{\mathbf{0}}$ & $\boldsymbol{c}_{\mathbf{3}}=\boldsymbol{c}_{\mathbf{4}}$ & $\boldsymbol{C}$ & $\boldsymbol{m}^{\mathbf{0}}$ & $\boldsymbol{r}^{\mathbf{0}}$ & $\boldsymbol{w}^{\mathbf{0}}$ \\
\hline 5 & 0,1400 & 98 & 2 & 1 & $\mathbf{1 0 0}$ & 0,3719 & 48 & 2 & 1 & 1 & 0,3456 & 40 & 2 & 1 \\
10 & 0,2239 & 68 & 2 & 1 & 575 & 0,4954 & 73 & 2 & 1 & $\mathbf{2}$ & 0,3719 & 48 & 2 & 1 \\
15 & 0,2999 & 56 & 2 & 1 & 1050 & 0,5967 & 93 & 2 & 1 & 3 & 0,3947 & 55 & 2 & 1 \\
$\mathbf{2 0}$ & 0,3719 & 48 & 2 & 1 & 1525 & 0,6872 & 110 & 2 & 1 & 4 & 0,4150 & 61 & 2 & 1 \\
25 & 0,4414 & 43 & 2 & 1 & 2000 & 0,7709 & 125 & 2 & 1 & 5 & 0,4335 & 66 & 2 & 1 \\
\hline
\end{tabular}




\section{Conclusões e Sugestões}

Nos casos em que existem erros de classificação no sistema de inspeção por atributos, o modelo desenvolvido considerando o uso de classificações repetidas e um critério para o julgamento do item conforme proporciona uma estratégia de controle tão ou mais eficiente que os modelos de inspeção simples. O que determina o quão mais eficiente será o modelo desenvolvido é a combinação específica dos fatores de custo, das probabilidades de produção nos estados I e II, da probabilidade de mudança de estado e dos erros de inspeção do sistema de controle em questão. Em linhas gerais, quanto menor for o custo de uma inspeção $\left(c_{0}\right)$ e quanto maior a incidência dos erros Tipo I e II, maior será a diferença observada entre a estratégia obtida com o modelo deste trabalho e aqueles de inspeção simples. Para o exemplo numérico adotado, em que há erros de classificação da ordem de $1 \%$, a estratégia de classificações repetidas gera uma redução de $3,48 \%$ no custo médio do sistema de controle por item produzido e não descartado, quando comparado ao sistema de inspeção simples.

Para trabalhos futuros sugere-se a inclusão no modelo da decisão de descartar ou enviar para o mercado os itens que compõe o atraso e o item inspecionado. Propõe-se também o uso de uma amostra com dois ou mais itens. Esta modificação deve aumentar significativamente a complexidade do modelo, pois passa a existir a probabilidade de ocorrência da mudança de estado entre os itens que compõem a amostra.

\section{Agradecimentos}

Os autores agradecem a Fapesp, CNPq e Capes pelo apoio financeiro.

\section{Referências Bibliográficas}

(1) Borges, W; Ho, L.L. \& Turnes, O. (2001). An analysis of Taguchi's on-line quality monitoring procedure for attributes with diagnosis errors. Applied Stochastic Models in Business and Industry, 17, 261-276.

(2) Greenberg, B.S. \& Stokes, S.L. (1995). Repetitive testing in the presence of inspection errors. Technometrics, 37, 102-111.

(3) Nayebpour, M.R. \& Woodall, W.H. (1993). An analysis of Taguchi's on-line quality monitoring procedure for attributes. Technometrics, 35, 53-60.

(4) Quinino, R. \& Suyama, E. (2002). Número ótimo de classificações independentes com erro na avaliação da conformidade de produtos. Pesquisa Operacional, 22, 1-8.

(5) Quinino, R. \& Ho, L.L. (2004). Repetitive tests as an economic alternative procedure to control attributes with diagnosis errors. European Journal of Operation Research, 155, 209-225.

(6) Ross, S.M. (2003). Introduction to probability models. 8th edn. Academic Press, San Diego.

(7) Taguchi, G.; Elsayed, E.A. \& Hsiang, T. (1989). Quality Engineering in Production in Systems. McGraw-Hill, New York.

(8) Taguchi, G.; Chowdhury, S. \& Wu, Y. (2004). Taguchi's Quality Engineering Handbook. John Wiley \& Sons, Inc. New Jersey. 